Universitas, Volumen 2, Número 2, 2008,25-28, ISSN 2071-2573

๑ 2008 UNAN-León, Editorial Universitaria

\title{
Frecuencia de parásitos intestinales en expendedores de alimentos ubicados en los recintos de la UNAN-León
}

\author{
Diana Margarita Cortés Selva ${ }^{1}$, Martha Raquel Estrada Leytón'ㄹ, Karla Yessenia Areas Barrera ${ }^{1}$, Aleyda Téllez \\ Sierra $^{2^{*}}$
}

1. Escuela de Bioanálisis Clínico. Facultad de Ciencias Médicas. UNAN-León.

2. Departamento de Microbiología y Parasitología, Facultad de Ciencias Médicas, Universidad Nacional Autónoma de Nicaragua, León (UNAN-León).

\section{RESUMEN}

\begin{abstract}
Se realizó un estudio de corte transversal en expendedores de alimentos ubicados en los recintos de la UNAN-León para determinar la frecuencia de parásitos intestinales. Durante los meses de Mayo a Julio se estudiaron un total de 40 individuos a cada uno se le tomó una muestra fecal y se le realizó una encuesta para medir el grado de conocimiento sobre los mecanismos de transmisión de parásitos intestinales. Las muestras fueron analizadas en el laboratorio del departamento de Microbiología y Parasitología, Facultad de Ciencias Medicas, UNANLeón, utilizando el método con solución salina y lugol, y concentración de Ritchie. Los resultados demuestran una tasa de prevalencia global de parásitos intestinales del 38\%. Entamoeba coli fue el parásito de mayor prevalencia con un 39\%, seguido de Endolimax nana (22\%), Giardia lamblia (17\%), Entamoeba histolytica (11\%), Entamoeba hartmani $(5 \%)$, e Iodamoeba büstchilli (5\%). El nivel de conocimiento de la población sobre transmisión de Amebas y Ascaris lumbricoides fue relativamente satisfactorio en contraste con G. lamblia en el que la población conocía muy poco sobre las distintas formas de adquirir la infección.
\end{abstract}

Palabras claves: parásito intestinal, expendedores de alimentos, frecuencia, grado de conocimiento.

\section{INTRODUCCIÓN}

El parasitismo constituye un importante problema de salud pública por su elevada prevalencia y distribución universal. ${ }^{[1]}$ La Organización Mundial de la Salud(OMS) estima que más de 2 mil millones de personas en todo el mundo están infectados por parásitos intestinales.

El problema de salud sigue aumentando y es uno de los más persistentes de hoy. En general causan una significante morbilidad y mortalidad en el mundo, particularmente en regiones en desarrollo y en personas con diferentes grados de inmunocompetencia. ${ }^{[2]}$

La transmisión de parásitos intestinales usualmente ocurre debido a un mecanismo oral pasivo, a la ingesta de quistes y huevos, sobre todo por medio del agua, alimentos o manos contaminadas con residuos fecales. [3]

En la UNAN-León existen varios expendios de comida, siendo la venta de alimentos muy difundida. En la República de Nicaragua el MINSA cuenta con 252 inspectores sanitarios que se encargan de regular la distribución y venta higiénica de alimentos, un número insignificante de funcionarios en comparación con la cantidad de establecimientos existente en todo el territorio nacional. ${ }^{[4]}$ Siendo la prioridad los mercados se deja a un lado las inspecciones sanitarias a otros expendios de alimentos, por lo que se hace menester realizar estudios para evaluar la presencia de parásitos en los manipuladores de comidas de los comedores universitarios, pues no existe un programa serio de monitoreo sobre las medidas higiénico sanitarias de los mismos, siendo los manipuladores un medio principal de transmisión de parásitos y enfermedades.

\section{DISEÑO METODOLÓGICO}

El estudio fue de tipo descriptivo, corte transversal. Se llevó a cabo de Mayo a Julio del 2007 con expendedores de alimentos ubicados en los diferentes recintos de la UNAN-León.

Se examinaron a 40 trabajadores, a los que se le realizó un examen coproparasitológico y se les aplicó una encuesta para evaluar el conocimiento sobre parásitos intestinales y sus formas de transmisión y para conocer las medidas higiénicas que implementaban.

Las muestras fueron analizadas por solución salina y lugol (examen general de heces) y método de concentración de Ritchie. Estas fueron analizadas en la Sección de Parasitología del Laboratorio de Microbiologia de la Facultad de Ciencias Médicas de la UNAN- León. La información se analizó en SPSS 12.0. 


\section{RESULTADOS Y DISCUSIÓN}

Respecto a las prácticas higiénicas utilizadas por los trabajadores se encontró que la mayoría ( $80 \%)$ no usa guantes para preparar o servir los alimentos. Por otro lado, la mayoría refiere lavado de manos después de ir al inodoro $(70 \%)$ y lavado de verduras antes de la preparación de alimentos (97\%). En la mayoría de los establecimientos (83\%) predominó el uso de agua potable (grifo). (ver tabla 1)

Lo que refleja que aparentemente la mayoría de los expendedores cumplen con los requisitos básicos para evitar la transmisión de parásitos, lo que puede ser explicado en que actualmente se hace más énfasis en promover acciones que ayuden a preservar la salud. Sin embargo los resultados pueden ser subjetivos pues están en dependencia de la veracidad de la respuesta de los encuestados.

El número de clientes que cada manipulador atiende varía entre 50 personas a 780 . Siendo atendidos diariamente un promedio de 416 personas. (ver tabla 2)

Tabla 1. Distribución de Factores higiénicos-sanitarios de los manipuladores de alimentos de la UNAN-León

\begin{tabular}{|c|c|c|c|}
\hline \multicolumn{2}{|c|}{ Características higiénicas } & \multirow{2}{*}{$\begin{array}{c}\text { Frecuencia } \\
8\end{array}$} & \multirow{2}{*}{$\begin{array}{c}\text { Porcentaje } \\
\text { (\%) } \\
20\end{array}$} \\
\hline Uso de guantes & $\mathrm{Si}$ & & \\
\hline & No & 32 & 80 \\
\hline \multirow[t]{2}{*}{ Protección de móscas } & Si & 40 & 100 \\
\hline & No & 0 & 0 \\
\hline \multirow{4}{*}{ Lavado de vegetales } & Siempre & 39 & 97.5 \\
\hline & A veces & 1 & 2.5 \\
\hline & Si hay agua & 0 & 0 \\
\hline & Nunca & 0 & 0 \\
\hline \multirow[t]{2}{*}{ Suministro de agua } & Grifo & 33 & 82.5 \\
\hline & Depósito & 7 & 17.5 \\
\hline \multirow{3}{*}{$\begin{array}{l}\text { Lavado de manos } \\
\text { despues de ir al inodoro }\end{array}$} & Siempre & 28 & 70 \\
\hline & A veces & 12 & 30 \\
\hline & Si hay agua & 0 & 0 \\
\hline \multirow{2}{*}{$\begin{array}{l}\text { Lavado de manos antes } \\
\text { de preparación de } \\
\text { alimentos }\end{array}$} & $\mathrm{Si}$ & 100 & 100 \\
\hline & No & 0 & 0 \\
\hline
\end{tabular}

Tabla 2 . Promedio de clientes atendidos diariamente por cada manipulador de alimentos de la UNAN-León

\begin{tabular}{cccc}
\hline $\begin{array}{c}\text { No de clientes que } \\
\text { atiende }\end{array}$ & minimo & Máximo & promedio \\
\hline Clientes & 50 & 780 & 416.25
\end{tabular}

Tabla 3. Prevalencia general de parasitismo intestinal en manipuladores de alimentos de la UNAN-León

\begin{tabular}{ccc}
\hline $\begin{array}{c}\text { Parásitos intestinales } \\
\text { observados }\end{array}$ & Frecuencia & $\begin{array}{c}\text { Porcentaje } \\
\%\end{array}$ \\
\hline Si & 15 & 37.5 \\
No & 25 & 62.5 \\
\hline Total & 40 & 100.0 \\
\hline
\end{tabular}

Este resultado demuestra que la cantidad de personas expuestas a contaminación por microorganismos en general es sumamente elevada, por lo que se deben reforzar las medidas higiénicas y los controles sanitarios.

Se encontró una prevalencia general de parasitosis intestinal de $38 \%$ (ver tabla 3 ), una tasa relativamente alta si se toma en cuenta la población universitaria a la que atienden estos manipuladores y la cual puede resultar contaminada.

La especie de parásito que se encontró con mayor frecuencia fue Entamoeba coli (39\%) seguido de Endolimax nana (22\%), Giardia lamblia en un 17\%, Entamoeba histolytica (11\%) Entamoeba hartmanni e Iodamoeba büscthilii con $6 \%$ cada uno. (ver tabla 4)

Nuestros resultados son comparables a los reportados por Ana Port Lourenco en Brasil[ ${ }^{[5]}$ donde el parásito de mayor prevalencia en manipuladores de alimentos de hospitales públicos y privados fue también Entamoeba coli seguido de Endolimax nana. Los datos obtenidos se relacionan además con los resultados en Chile a manipuladores de alimentos en hospitales por Arias et a[ ${ }^{[6]}$, que reportó una frecuencia elevada de Endolimax nana $(46.6 \%)$, seguido de E. coli $(41.2 \%)$ y Entamoeba histolytica (12.1\%).

Así también en Cuba por Gómez et $a^{[1]}$, donde la prevalencia de población parasitada fue de $29.4 \%$ y el parásito patógeno más frecuente fue Giardia lamblia. A pesar de que la Entamoeba coli no es patógena y no tiene gran impacto en la clínica, si es de suma importancia su reporte de Laboratorio dado que el estudio es realizado en manipuladores de alimentos y es de relevancia epidemiológica ya que muestra que el 
Tabla 4. Distribución de especie de parásito en muestras fecales positivas de manipuladores de alimentos de la UNAN-León

\begin{tabular}{lcc}
\hline \multicolumn{1}{c}{ Tipo de parásito } & Frecuencia & $\begin{array}{c}\text { Porcentaje } \\
\%\end{array}$ \\
\hline E.coli & 7 & 38.8 \\
E.nana & 4 & 22.2 \\
E. histolytica & 2 & 11.1 \\
Iodamoeba butschlii & 1 & 5.5 \\
Giardia lamblia & 3 & 16.6 \\
E.hartmanni & 1 & 5.5 \\
\hline \multicolumn{1}{c}{ Total } & 18 & 100 \\
\hline
\end{tabular}

Tabla 5. Tasa de poliparasitismo encontrada en expendedores de alimentos de la UNAN-León

\begin{tabular}{lcc}
\hline No de parásito & Frecuencia & $\begin{array}{c}\text { Porcentaje } \\
\%\end{array}$ \\
\hline Un parásito & 12 & 80 \\
Dos o más parásitos & 3 & 20 \\
\hline Total & 15 & 100 \\
\hline
\end{tabular}

individuo parasitado puede estar expuesto a otros tipos de parásitos y también ser una vía de transmisión de una variedad de éstos.

Se encontró una tasa de poliparasitismo del $20 \%$ (ver tabla 5), de suma importancia pues muestra el grado de contaminación a la cual el individuo ha estado expuesto.

En cuanto al conocimiento que estos manipuladores poseían sobre parásitos se encontró que todos habían escuchado hablar de ellos, pero sólo el $40 \%$ pudo mencionar algún parásito (ver tabla 6). De éstos, los más conocidas son Ascaris lumbricoides (lombrices).

Aunque todos los encuestados han escuchado sobre parásitos, el grado de conocimiento es pobre pues una minoría pudo nombrar algún nombre de un tipo específico de parásito, esto puede ser debido a que el grado de escolaridad en la población estudiada era bajo.

Siendo G. lamblia uno de los parásitos encontrados con mayor frecuencia y que sus formas de transmisión están muy ligadas a la manipulación de alimentos y calidad del agua, al evaluar el nivel de conocimiento de los expendedores encontramos que solamente el $40 \%$ tenía parcialmente una opinión acertada al afirmar que el parásito se adquiere vía fecal-oral. En el caso de Amebas y A. lumbricoides la información contrasta en el sentido que la mayor parte de individuos conoce al menos una forma de transmisión.
Tabla 6. Conocimiento general sobre parásito que poseen expendedores de alimentos de la UNAN-León

\begin{tabular}{lcc}
\hline \multicolumn{1}{c}{ Parásito mencionado } & Frecuencia & $\begin{array}{c}\text { Porcentaje } \\
\%\end{array}$ \\
\hline No mencionó & 24 & 60.0 \\
Amebas & 3 & 7.5 \\
Lombrices & 9 & 22.5 \\
Lombrices y amebas & 1 & 2.5 \\
Tricocéfalos & 2 & 5.0 \\
Tricocefalos, amebas & 1 & 2.5 \\
y lombrices & & \\
\hline \multicolumn{1}{c}{ Total } & 40 & 100 \\
\hline
\end{tabular}

\section{CONCLUSIONES}

El parasitismo intestinal se detectó en el $38 \%$ de los manipuladores de alimentos de la UNAN-León, siendo el más frecuente E. coli, seguido de E. nana. El parásito patógeno más frecuente fue G. lamblia. Se encontraron casos de poliparasitismo, detectándose una tasa del $20 \%$.

Mientras que las costumbres higiénicas- sanitarias entre los manipuladores de alimentos son relativamente satisfactorias. Sin embargo, se necesitan hacer esfuerzos en extremar otras medidas de higiene como el uso de guantes, pues esta es una vía de importancia en la transmisión de parásitos.

El parásito más conocido entre los expendedores de alimentos es Ascaris lumbricoides, llamado popularmente "lombrices". El nivel de conocimiento de la población fue bajo con relación al modo de transmisión de G. lamblia, en cambio fue satisfactorio en cuanto al conocimiento de transmisión de Amebas y A. lumbricoides.

\section{REFERENCIAS BIBLIOGRÁFICAS}

1. GOMEZ VITAL M. N. (1999) Parasitismo intestinal en manipuladores de alimentos. Rev Cubana Med Gen Integr, sep.- oct. , vol.15, no.5, p.520-523.

2. RUBIO, J. M. Parasitosis intestinales humanas más frecuentes en España. Centro Nacional de Microbiología España.

3. TAPELLA F. A. Parasitología (monografía en Internet). Universidad Nacional de Rosario, Argentina. (acceso en Abril 2007). Disponible en: http://www.monografias.com/trabajos38/ parasitologia/parasitologia.shtml 
4. ENRIQUEZ O., COLLADO R. Somos un pueblo desaseado. El Nuevo Diario Online. Domingo 8 de Abril del 2001. Managua, Nicaragua. Disponible en: http://archivo.elnuevodiario.com.ni/2001/abril/08abril-2001/especiales/especiles1.html

5. PORT A., OCHOA C. (2004) Machado O. Hospital food handles in Niteroi,RJ, Brazil: intestinal parasitism. ALAN, Diciembre, vol 54, no.4, p 395401.

6. ARIAS B., SOTO E. (1987) Sepúlveda L, Herrera A. Infecciones intestinales por parásitos y/o comensales en manipuladores de alimentos de hospitales del sector norte de Santiago, Chile. Boletín chileno de Parasitología; 42:84-86. 